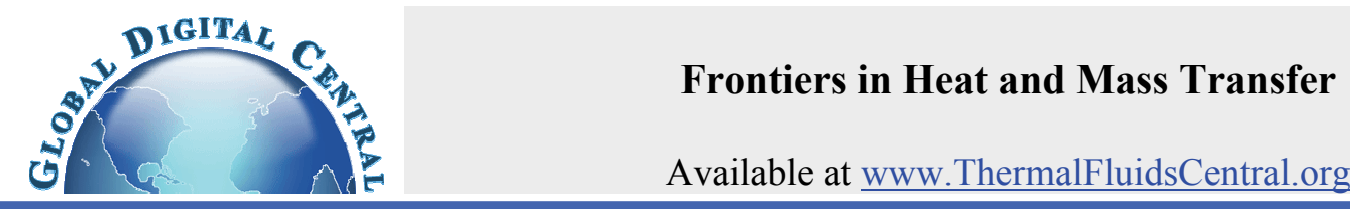

\title{
DETERMINING HEAT TRANSFER COEFFICIENT OF HUMAN BODY
}

\author{
A. Najjaran", Ak. Najjaran, A. Fotoohabadi, A.R. Shiri \\ Islamic Azad University Branch of Shiraz, Shiraz, Fars, Iran, 7154845589, Iran
}

\begin{abstract}
In this paper, the aim is obtaining convection coefficient of human body. This field of study is essential in study of ventilation systems, astronauts' clothes and any other fields in which human body is the main concern. At first a 3D human body has been designed by unstructured grids. Feet and hands are stretched completely in considered sample. Two postures (standing and supine) are considered for body. Soles and the back of entire body are considered in contact with the ground respectively in these postures. Other parts of human body are exposed to surrounding air. The heat transfer and the body temperature are assumed steady and constant. The results are obtained by applying finite volume method for each grid and extracted by the weighted area method in Fluent ${ }^{\mathbb{R}}$. Then the attained results are validated with the recent experimental results. Good agreement is observed between the obtained results and the previous experimental results. Finally two formulas are derived for natural convection coefficient of human body.
\end{abstract}

Keywords: convective heat transfer coefficient, standing, supine, human body, numerical simulation.

\section{INTRODUCTION}

Natural convection is a process which doesn't need any external force to complete the thermal cycle. For instance, if a heat source is assumed in a cooler space, the surrounding air which is close to the heat source is warmed and expanded. So because of the decrease of its density the air goes upward. Then in consequence of vicinity with cooler air, its temperature decreases and it compresses again and therefore it goes back to its initial position in the cycle. This process takes place only because of the temperature difference between the heat source and surrounding air. This paper is aimed at finding the natural convection coefficient of human body. This coefficient is essential for determining the exchanged convectional heat between human body and surrounding air.

In previous papers convection coefficient of human body is determined by different methods. The reported convection coefficients are different and contain questionable accuracies. The reasons of differences in reported coefficients are various. Firstly, the natural convection coefficient is affected by gravity force. The effect of gravity force direction is different in various body postures and would cause different results. Secondly, human body sample shape causes an important effect. Complicated body models increase airflow disturbance and so the flow disturbance around the body should be analyzed. Thirdly, as Kurazumi et al. mentioned, 10 to $20 \%$ of body surface doesn't participate in convectional heat transfer (for example because of ground contact) but in the majority of recent researches convectional heat transfer is considered in all entire body surface (area ratio 1) for simplicity. Fourthly, in experimental studies unlike analytical methods, measuring quantities has some errors. Fifthly, experimental studies are with some limitations which decrease the accuracy of results. For example obtaining a specific thermal condition in laboratory is difficult or convectional heat should be analyzed in plastically mannequins. Furthermore numbers of sensors which are used in experimental researches are considered as disturbing factor and should be limited too. For example, the convection coefficient in different members is different; so this parameter should be measured for each member separately. But the applied sensors are considered as disturbing structures in heat transfer and should be limited. In some studies, the radiated heat is calculated and then is subtracted from the total heat for determining convection coefficient. The radiation heat depends on angle factor.

By considering the 3D body of human, the angle factor has various amounts and so it causes different heat radiation. Therefore the calculated quantities for convection heat and convection coefficient don't have proper accuracy. In this paper, natural convection coefficient of human body is calculated for two usual postures (supine \& standing). Of course these postures are samples and other postures can be analyzed too. Despite of previous studies skin and meat is considered for the body. The areas which have no convection heat transfer with surroundings are calculated and subtracted from the total area. The assumed area of the body is optional and so it doesn't have the limitations of the previous methods. Although the area of the actual heat transfer surfaces might be less or more than the considered amount, but because the convection heat transfer coefficient is calculated per unit area so the errors can be neglected. Unknown parameters are determined from the weighted area method. The obtained results are validated with recent experimental results. Finally two formulas are obtained from the results.

\section{GOVERNING EQUATIONS}

Heat transfer of body with surrounding air can be divided into four main methods of Convection, Conduction, Radiation and Evaporation. Convection heat flux of human body follows conjugate equations because of having a specific structure similar to fins. Human body is the same as six cylindrical fins and for each part the formula of the cylindrical fins is used for calculating its heat flux. Of course in some studies human body is considered as a collection of one spherical and five cylindrical fins. By considering a steady state heat transfer and

\footnotetext{
* Corresponding Author Email: anajaran@iaushiraz.ac.ir
} 
applying these equations for human body, the natural convection coefficient and also convection heat flux are determined.

Equations (1) to (3) show the continuity, momentum and energy governing equations. These equations can be used in the conditions in which the viscosity is constant or zero.

$$
\frac{D \rho}{D t}=\frac{\partial \rho}{\partial t}+V \cdot \nabla \rho=-\rho \nabla \cdot V
$$

$\rho \frac{D V}{D t}=\rho\left(\frac{D V}{D t}+V . \nabla V\right)=F-\nabla P+\mu \nabla^{2} V+\frac{\mu}{3} 3 \nabla(\nabla . V)$

$$
\rho C_{p} \frac{D T}{D t}=\rho C_{p}\left(\frac{D T}{D t}+V . \nabla T\right)=\nabla \cdot(k \nabla T)+q^{\prime \prime \prime}+\beta T \frac{D T}{D t}+\mu \phi_{v}
$$

where $\mathrm{V}$ is the velocity vector, $\mathrm{T}$ is the surrounding temperature, $\mathrm{t}$ is the time, $\mathrm{F}$ is volumetric force per unit volume, $C_{p}$ is specific heat capacity in constant pressure, $\mathrm{P}$ is static pressure, $\rho$ is fluid density, $\beta$ is thermal expanding coefficient of fluid, $\phi_{v}$ is viscosity losses (which is irreversible part of heat transfer because of the viscosity forces) and $q^{\prime \prime \prime}$ is the heat generation per unit volume. The thermal expanding coefficient of fluid is in $\beta=-(1 / \rho)(\partial \rho / \partial T)_{P}$ which the symbol of $\mathrm{P}$ denotes the constant pressure. For a Noble gas this parameter is equal 5 to $\beta=1 / T$ which $\mathrm{T}$ is the absolute temperature in terms of Kelvin. $D / D t$ is the total or partial differential which might become apparent as $(\delta(\nabla V) / \delta T)$. The main factor for natural convection heat transfer is temperature differences. Warming and cooling processes of body by environmental air (or in fact temperature differences) causes environmental air density changes. Density variations and buoyancy forces result in fluid movement which causes natural convection. For example, during cooling process of body, the vicinity air gets warm and so its volume increases and its density decreases and so it goes upward.

\section{SOLUTION PROCEDURE}

At first a sample of human body is designed by Solid works software and then its grids are generated by Gambit software. The final designed model is transferred to fluent software. Boundary conditions (such as body temperature, air density, surrounding temperature and pressure) are set as inputs. The software uses Boussinesq and boundary layer approximations for solving the problem. The initial guesses are obtained by software and the desired accuracies of results of governing equations (Equations (1) To (3)) are defined by users. By convergence of the problem the considered parameters are determined in each grid. Finally the outputs are extracted by use of weighted area method.

\section{BOUSSINESQ AND BOUNDARY LAYER APPROXIMATIONS}

The governing equations of natural convection are dependent on each other and have elliptical and partial differential forms. Unwished viscosity deviations which are caused by the temperature changes also cause calculations to be in a conjugate form. Generally some methods are used to make these equations simple which in these methods Boussinesq and boundary layer approximations are very important. These approximations are widely used for natural convection. An important condition for validation of these approximations is $\beta(\mathrm{T}-\mathrm{T} \infty)<1$. When the temperature differences are low this simplification is valid. In Boussinesq approximation the density deviations are neglected. Moreover density differences of the fluid usually are estimated by temperature effect (the effect of pressure is neglected). The density differences are estimated for the thermal buoyancy by Equation (4).

$$
\rho_{\infty}-\rho=\rho \beta\left(T-T_{\infty}\right)
$$

The other method is boundary layer approximation. In natural convection, the velocity out of the boundary layer is very low and is affected with the surrounding pressure. The surrounding pressure is the hydrostatic pressure. In addition increasing of heat or mass of the fluid is assumed to be limited to thin layer close to the body surface. So it is assumed that the gradients in the tangent direction of the surface are very smaller than the ones that are in the normal (perpendicular) direction. The main result of boundary layer approximation is that the flow terms (except the vertical ones) are neglected in momentum and heat equations. These assumptions are losing their validity in unsteady state heat transfer. Therefore in this paper the ground temperature is assumed the same as surrounding temperature.

\section{INITIAL CONDITION AND RESULTS' CONVERGENCY}

There is no general law for convergence. So each problem has its specific technique. As is said in the natural convection problems the flow velocity is low. Viscosity deviations cause considerable conjunction too. So for convergence of the results, at the beginning of the solving velocities are assumed close to zero and In addition heat transfer is assumed steady. Then for several iterations the energy equations are solved for determining a better initial guess. After that, the continuum and momentum equations are solved with energy equation simultaneously. Then the flow velocity is increased to determine the flow treatment. This performance is done by increasing the gravity acceleration. After obtaining the magnified flow treatment, the gravity acceleration is decreased in several consecutive processes until real amounts are attained.

\section{MATHEMATICAL DEFINITION OF WEIGHTED AREA METHOD}

The weighted area average (or in fact weighted mean) of a non-empty set of convection coefficients

$$
\left\{h_{\text {conv }, 1}, h_{\text {conv }, 2}, \ldots, h_{\text {conv }, n}\right\}
$$

With non-negative weights

$$
\left\{w_{1}, w_{2}, \ldots, w_{n}\right\}
$$

Is the quantity

$$
\bar{h}_{\text {conv }}=\left(\sum_{i=1}^{n} w_{i} h_{\text {conv }, i}\right) /\left(\sum_{i=1}^{n} w_{i}\right)
$$

Therefore convection coefficients of grids with a high weight contribute more to the weighted area than do convection coefficients of grids with a low weight. The weights cannot be negative. Some may be zero, but not all of them because division by zero is not allowed. The formulas are simplified when the weights are normalized such that they sum up to 1 , i.e. $\sum_{i=1}^{n} w_{i}=1$. For such normalized weights the weighted area is simply $\bar{h}_{\text {conv }}=\sum_{i=1}^{n} w_{i} h_{\text {conv }, i}$.

\section{RESULTS AND DISCUSSIONS}

In the previous experimental studies air temperature, relative humidity and flow velocity are measured using an Assmann aspiration Psychrometer and a non-directional hot bulb-type anemometer. The 
vertical air temperature profile and the temperatures of all room wall surfaces are also taken with T-type thermocouples. Considering the effects of relative humidity and the other parameters on the natural convection coefficient, in this paper these parameters are set as inputs and so it is no need of using these devices. Skin temperature, conduction coefficient, specific heat capacity and average density of human body are considered $308 \mathrm{~K}, 0.209 \mathrm{~W} / \mathrm{mK}, 3470 \mathrm{~J} / \mathrm{KgK}$ and $1000 \mathrm{~kg} / \mathrm{m}^{3}$, respectively. In addition, the air pressure is considered $101325 \mathrm{~Pa}$. The surrounding is considered uniform and the heat transfer is assumed steady. The remaining fluid characteristics in natural convection are considered constant. The considered human body exchanges heat just with the air. The local differences of the flow velocity in lower and upper part of body are neglected and the whole body is exposed to natural convection with the same characteristic. As is said in the introduction the calculations based on total heat have some problems. Therefore in this paper, calculations are done based on the convection total heat.

One of the differences between the present work and the previous ones is that the body is organized from a large number of approximating surfaces. For each part surface area or area ratio is determined. So the area ratios of recent researches that were related to limited number of surfaces are not used. A human body sample includes 1874427 approximating surfaces, 191524 boundary nodes and 896726 cells in standing posture. Also it contains 1572162 approximating surfaces, 143830 boundary nodes and 771108 cells in supine posture (see Figure 1). Grids are considered unstructured and the cells are considered tetrahedron.

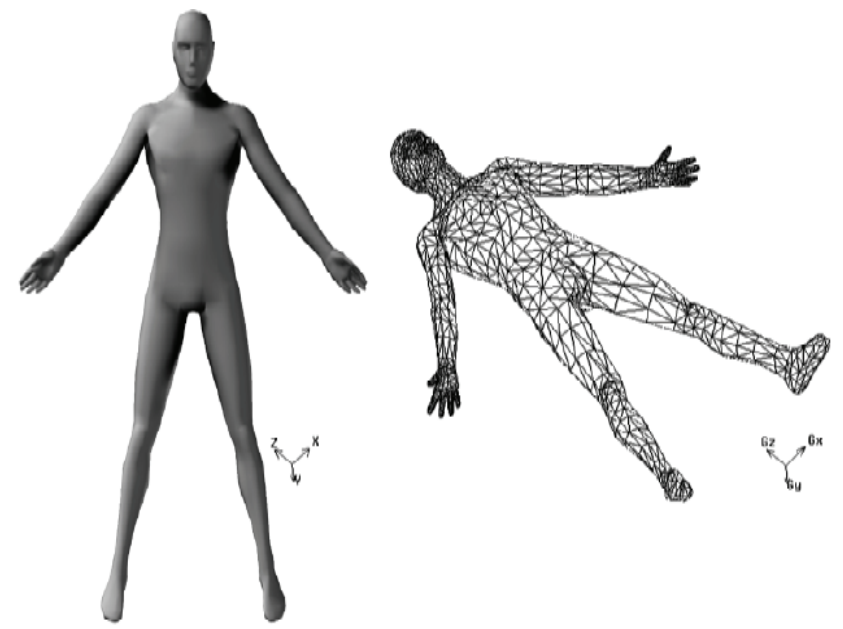

Fig. 1 Human Body model (standing and spine postures)

As shown in Figs. 2 and 3 concentration of grids is more around the body. This concentration leads to more accurate results and decreases the number of grids. The reasons of the excess number of grids and concentration of grids around the body are the complex structure of body and conjugation of thermal heat exchange around the body. Body grid generation is done in such a way that different parts of body have no contact with each other. For example fingers don't have any contact with each other. Figure 2 shows the grid test of analyzed samples.

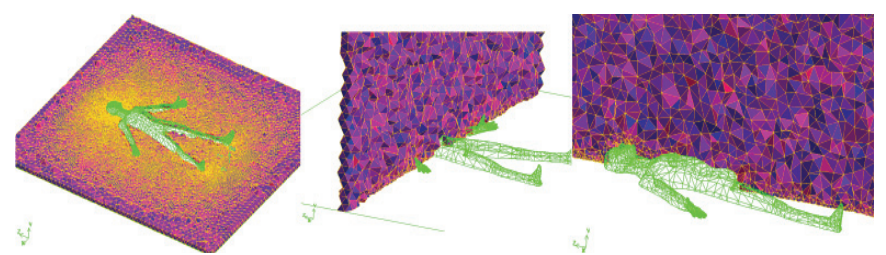

Fig. 2 Mesh gird test in different directions

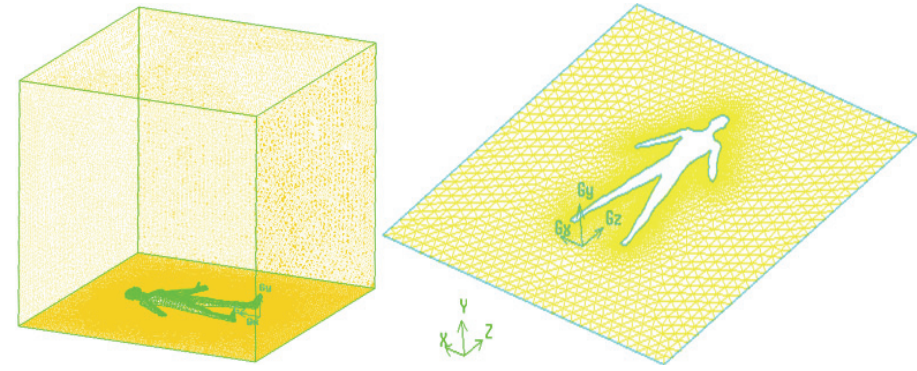

Fig. 3 Analyzed domains and meshed model in supine posture

A domain with dimension of $2 \times 2 \times 2$ meter is considered around the body (see Figure 3 ). This domain is produced in such a way that in standing posture soles and in supine posture the whole back of the body are in contact with the ground. It is limited from the ground and from the other side is exposed to the air. The ground and the air are in a thermal balance and are considered to have the same temperature.

After calculating the convection coefficient for each grid, the average convection coefficient is determined for the entire body. For determining the average of obtained results several computational methods exist such as actual area average, actual vertex average, standard deviation and the weighted area average. In this paper, the results are determined based on the last two methods. By comparing the results it is concluded that the weighted area average method has a better performance in determining average between unbalanced parameters. Due to size differences of surfaces using standard deviation method faces with problem. Table 1 shows some obtained results for standing posture by the use of standard deviation method (the temperatures are in Kelvin and the convection coefficient unit is $\mathrm{W} / \mathrm{m}^{2} \mathrm{~K}$

Table 1

\begin{tabular}{c|ccc}
\hline Case & $\begin{array}{c}\text { Sur. } \\
\text { Tem. }\end{array}$ & $\begin{array}{c}\text { Body } \\
\text { Tem. }\end{array}$ & $\begin{array}{l}\text { Conv. } \\
\text { Coef. }\end{array}$ \\
\hline 1 & 295 & 304 & 3.563 \\
2 & 293 & 298 & 3.715 \\
\hline
\end{tabular}

The histogram diagram of the results obtained by standard deviation method (see Fig. 4) shows the wide dispersal of the natural convection coefficient.

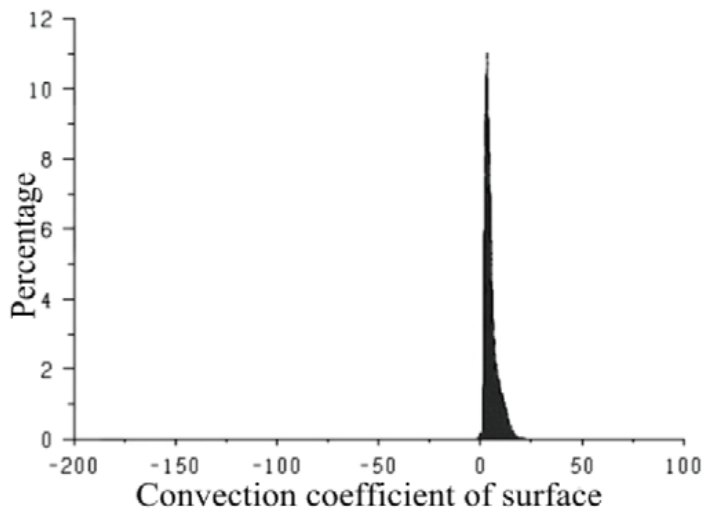

Fig. 4 Histogram diagram of convection coefficient obtained by standard division method

According to the statistical data, the most percentage of the outputs is close to the coefficients which are expressed in Table 1. The obtained results by weighted area method are shown in Table 2 It shows six 
cases which are considered for two standing and supine postures. In Table 2 Sur. Tem. denotes surrounding temperature and Re. Er. denotes relative error. The temperatures are in Kelvin and the convection coefficients are in $\mathrm{W} / \mathrm{m}^{2} \mathrm{~K}$.

Table 2

\begin{tabular}{c|ccccccc}
\hline $\begin{array}{c}\text { Sur. } \\
\text { Tem. }\end{array}$ & $\begin{array}{l}\text { Skin } \\
\text { Tem. }\end{array}$ & $\begin{array}{c}\text { Standined } \\
\text { Stand } \\
\text { Coef. }\end{array}$ & $\begin{array}{c}\text { Standing } \\
\text { Coef. Eq. (6) }\end{array}$ & $\begin{array}{c}\text { Re Er } \\
\%\end{array}$ & $\begin{array}{c}\text { Obtained } \\
\text { Supine } \\
\text { Coef. }\end{array}$ & $\begin{array}{c}\text { Supine } \\
\text { Coef } \\
\text { Eq. (7). }\end{array}$ & $\begin{array}{c}\text { Re } \\
\%\end{array}$ \\
\hline 289 & 308 & 3.3 & 3.29 & 0.41 & 2.03 & 2.60 & 22.02 \\
291 & 308 & 3.18 & 3.16 & 0.57 & 2.54 & 2.50 & 1.63 \\
293 & 308 & 3.09 & 3.03 & 2.06 & 2.4 & 2.39 & 0.56 \\
295 & 308 & 2.90 & 2.88 & 0.66 & 2.28 & 2.26 & 0.69 \\
297 & 308 & 2.65 & 2.72 & 2.52 & 2.03 & 2.13 & 4.65 \\
299 & 308 & 2.24 & 2.54 & 11.66 & 1.78 & 1.98 & 10 \\
\hline
\end{tabular}

In Kurazumi et al. formulas are derived for convection coefficients of supine and standing postures from experimental results. Equations (6) and (7), represent the obtained formulas for standing and supine respectively.

$h_{\text {supine }}=1.183 \Delta T^{0.347}$

$h_{\text {supine }}=0.881 \Delta T^{0.368}$

Table 2 also represents the relative errors for each case. These errors are calculated by comparing the obtained results with the results which are attained by Equations (6) and (7). The mean relative errors of the results related to standing and supine postures are, $2.98 \%$ and $6.59 \%$ respectively.

By using the obtained results of numerical simulation, two formulas are achieved for standing and supine postures. Equation (8) represents the obtained formula for standing.

$h_{s \tan \text { ding }}^{0.5}=1.951-16535(\operatorname{Ln}(\Delta T)) / \Delta T^{2}$

where $\mathrm{h}\left(\mathrm{W} / \mathrm{m}^{2} \mathrm{~K}\right)$ is the convection coefficient and $\Delta T$ is the temperature difference of the skin and surrounding. Equation (9) represents the convection coefficient for supine posture.

$$
h_{\text {supine }}{ }^{0.5}=1.734-15.016(\operatorname{Ln}(\Delta T)) / \Delta T^{2}
$$

Equations (8) and (9) are valid when the temperature difference is not high ( e.g. no bigger than 40 degree). Figure 5 shows the results which are reported by Hard and Dubois, Nielsen and Pedersen, Ohmoto and Mochida, Kurazumi et al., Nishi and Gagge and Najjaran et al. for convection coefficient of standing and supine postures. As is observed in Figure 5 By decreasing of the temperature difference, the convection coefficient also decreases. When the air temperature decreases, body decreases the skin temperature to prevent the extreme thermal losses. By decreasing the temperature difference between bodies and surrounding, the flow velocity also decreases. Consequently the coefficient of convection heat transfer decreases. Also it is observed that the convection coefficient in the standing posture is continuously more than the sitting posture (see Figure 5). Good agreement is seen between the obtained curves and Kurazumi et al. results.

By comparing the obtained results of all cases it is conducted that by increasing the temperature difference between body and surrounding air, the velocities in both postures increases layer by layer.

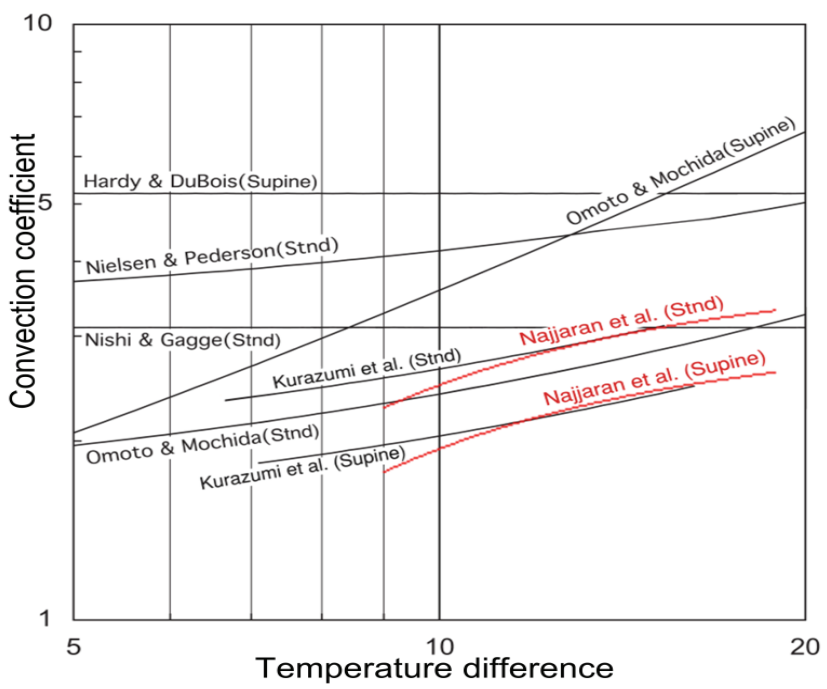

Fig. 5 Comparison of the obtained natural convection coefficient with the ones which are obtained by experimental methods in recent studies

In Fig. 5 the convection coefficients of postures which don't have any contact with the ground are not analyzed. These postures are unusual and are considered only because of experimental limitations (such as preventing the contact of body surfaces with each other [52]). Velocity path lines are factors which show the velocity gradient of air around human. Figures 6 and 7 show velocity contours around the body in standing and supine postures in two cases.

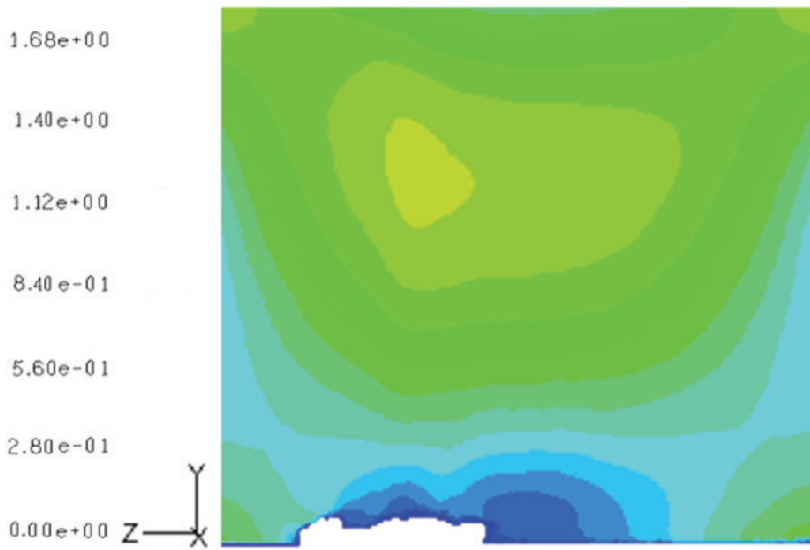

Fig. 6 Contour velocities in supine posture
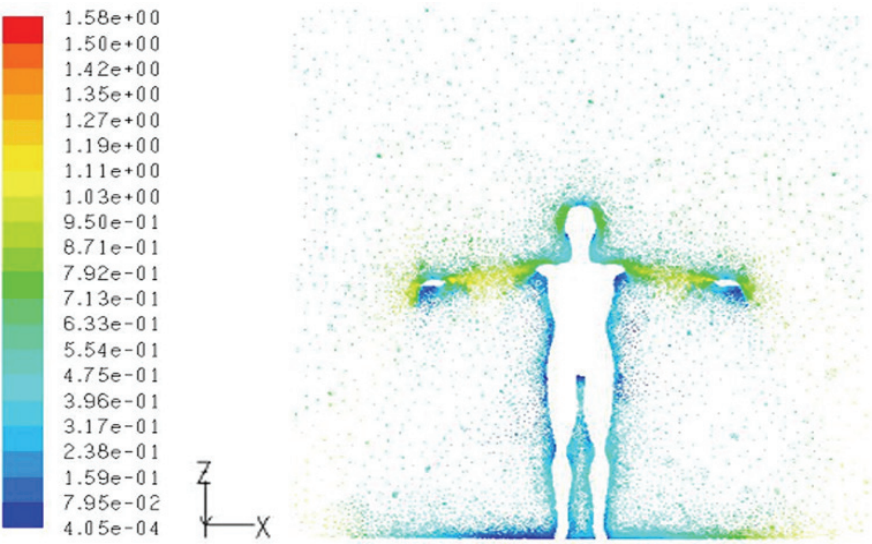

Fig. 7 Contour velocities in standing posture 


\section{CONCLUSIONS}

The aim of this research is determining of natural convection coefficient of human body which has many applications in different fields. A sample of human body was designed by unstructured grids. Two different postures (standing and supine) were considered for sample. Some parts of the body were in contact with the ground in these postures. The rest of surfaces of the body were exposed to the surrounding air. For determining results the weighted area method was used. Two formulas were reported for the considered postures for natural convection coefficient. The obtained results were compared with the experimental results of previous researches and their errors were calculated. Good agreement was observed between the obtained results and the previous experimental results. No need of experimental setup, fast determination of the coefficient in various conditions and accuracy of the results are the benefits of applying this method.

\section{NOMENCLATURE}

$\begin{array}{ll}C & \text { specific heat } \\ F & \text { volumetric force (per unit volume) } \\ h & \text { convection coefficient } \\ P & \text { static pressure } \\ q & \text { Heat generation } \\ t & \text { time } \\ T & \text { surrounding temperature } \\ V & \text { velocity vector } \\ w & \text { surface weight }\end{array}$

\section{Greek Symbols}

$\begin{array}{ll}\beta & \text { Thermal fluid expanding coefficient } \\ \rho & \text { fluid density }\left(\mathrm{kg} / \mathrm{m}^{3}\right) \\ \phi_{v} & \text { Viscosity losses }\end{array}$

\section{Subscripts}

$p \quad$ constant pressure

\section{REFERENCES}

Bejan, A., 2002, Convection Heat Transfer, Wiley \& Sons, Hoboken, NJ.
Hardy, JD., Dubois, EF., 1938, “The Technic Of Measuring Radiation And Convection," Journal of Nutrition, 15(5), 461-475.

Kurazumi, Y., Tsuchikawa, T., Ishiia, J., Fukagawaa, K., Yamatoc, Y., Matsubarad, N., 2008, "Radiative and Convective Heat Transfer Coefficients of the Human Body in Natural Convection," Building and Environment, 43, 2142-2153.

http://dx.doi.org/10.1016/j.buildenv.2007.12.012

Kurazumi, Y, Tsuchikawa, T, Yamato, Y, Kakutani, K, Matsubara, N, Horikoshi, T, 2003, "The Posture and Effective Thermal Convection Area Factor of the Human Body," Japanese Journal of Biometeology, 40(1), 3-13.

Kurazumi, Y, Tsuchikawa, T, Matsubara, N, Horikoshi, T, 2004, "Convective Heat Transfer Area of the Human Body," European Journal of Applied Physiology, 93(3), 273-85.

http://dx.doi.org/10.1007/s00421-004-1207-1

Mochida, T., 1977, "Convective And Radiative Heat Transfer Coefficients For Human Body," Transactions of AIJ, 258, 63-69.

Mochida, T., 1977, "Mean Skin Temperature Weighted with Both Heat Transfer Coefficient and Skin Area," Transactions of AIJ; 259,67- 73.

Mochida, T, Nagano, K, Shimakura, K, Kuwabara, K, Nakatani, T, Matsunaga, K, 1999, "Characteristics Of Convective Heat Transfer Of Thermal Manikin Exposed In Air Flow From Front," Journal of Human and Living Environment, 6(2), 98-103.

Nielsen, M, Pedersen, L., 1952, "Studies on the Heat Loss by Radiation and Convection from the Clothed Human Body," Acta Physiologica Scandinavica, 27, 272-94.

http://dx.doi.org/10.1111/j.1748-1716.1953.tb00943.x

Nishi, Y, Gagge, AP., 1970, "Direct Evaluation of Convective Heat Transfer Coefficient by Naphthalene Sublimation," Journal of Applied Physiology, 29(6), 830-8.

Ohmoto, Y., Mochida, T., 1995, "Influence of Posture upon Convective Heat Transfer Coefficient for the Human Body," Proc. of the 19th symposium on human-environment system, Kyoto, Japan; 25-34. 